\title{
Are Wolf-Rayet Stars Able to Pollute the Interstellar Medium of Galaxies? Results from Integral Field Spectroscopy
}

\author{
Enrique Pérez-Montero, ${ }^{1}$ Carolina Kehrig, ${ }^{1}$ Jarle Brinchmann,, \\ José M. Vílchez, ${ }^{1}$ Daniel Kunth, ${ }^{3}$ and Florence Durret ${ }^{3}$ \\ ${ }^{1}$ Instituto de Astrofísica de Andalucía-CSIC, Apartado. 3004, 18008 Granada, Spain \\ ${ }^{2}$ Leiden Observatory, Leiden University, P.O. Box 9513, 2300 RA Leiden, The Netherlands \\ ${ }^{3}$ Institut d'Astrophysique de Paris, UMR 7095 CNRS, Université Pierre \& Marie Curie, 98 bis boulevard Arago, 75014 Paris, France
}

Correspondence should be addressed to Enrique Pérez-Montero; epm@iaa.es

Received 17 May 2013; Accepted 30 August 2013

Academic Editor: Polychronis Papaderos

Copyright (C) 2013 Enrique Pérez-Montero et al. This is an open access article distributed under the Creative Commons Attribution License, which permits unrestricted use, distribution, and reproduction in any medium, provided the original work is properly cited.

\begin{abstract}
We investigate the spatial distribution of chemical abundances in a sample of low metallicity Wolf-Rayet (WR) galaxies selected from the SDSS. We used the integral field spectroscopy technique in the optical spectral range ( $3700 \AA-6850 \AA$ ) with PMAS attached to the CAHA $3.5 \mathrm{~m}$ telescope. Our statistical analysis of the spatial distributions of $\mathrm{O} / \mathrm{H}$ and $\mathrm{N} / \mathrm{O}$, as derived using the direct method or strong-line parameters consistent with it, indicates that metallicity is homogeneous in five out of the six analysed objects in scales of the order of several kpc. Only in the object WR404 is a gradient of metallicity found in the direction of the low surface brightness tail. In contrast, we found an overabundance of N/O in spatial scales of the order of hundreds of pc associated with or close to the positions of the WR stars in 4 out of the 6 galaxies. We exclude possible hydrodynamical causes, such as the metal-poor gas inflow, for this local pollution by means of the analysis of the mass-metallicity relation (MZR) and mass-nitrogen-to-oxygen relation (MNOR) for the WR galaxies catalogued in the SDSS.
\end{abstract}

\section{Introduction}

Wolf-Rayet (WR) galaxies host very bright episodes of star formation characterized by the emission of broad WR bumps in their optical spectrum [1]. The two main bumps in the optical range are the blue bump, centered at a wavelength of $4650 \AA$, produced by the emission from N v, N III, C III/C IV, and $\mathrm{He}$ II, and associated with WC and WN stars, and the red bump which is fainter, centered at $\sim 5800 \AA$, produced mainly by $\mathrm{C}$ III and C IV, and associated with WC stars. The lines making up these bumps originate in the dense stellar winds from WR stars ejecting metals into the interstellar medium (ISM).

The number of known WR galaxies has tremendously increased from the discovery of the first one (He 2-10: [2]), with different published catalogs [3-6], until the list of WR galaxies in the Sloan Digital Sky Survey (SDSS) by Brinchmann et al. [7] with around 570 objects with the identification of the WR bumps in their integrated spectra.
There is increasing evidence that the most challenging problems for this kind of objects appear in the low metallicity galaxies. Although it is well documented that the number of WR stars and the intensity of the WR bumps are higher for higher metallicities [8], the values found in some low metallicity H II galaxies, such as IZw18 [9], are claimed to be much higher than those predicted by synthesis population models (e.g., [10]).

Among the other important open issues regarding WR galaxies is the chemical enrichment of the ISM surrounding the stellar clusters where the WR stars are located. It is well known that there is an overabundance of the $\mathrm{N} / \mathrm{O}$ ratio found in some WR nebulae (e.g., [11-13]) and also in the ISM of some WR galaxies, where the WR features are diluted (e.g., HS0837 + 4717, [14], NGC5253, [15], other H II galaxies in [16, 17], and green pea galaxies, [18]). Brinchmann et al. [7] also showed that the median N/O ratio in WR galaxies with $\operatorname{EW}(\mathrm{H} \beta)<100 \AA$ has an excess of $\sim 25 \%$ in relation to the other star-forming galaxies in the SDSS. Chemical evolution 
TABLE 1: The sample of observed Wolf-Rayet galaxies with different properties, including names, redshifts, positions, WR index, and date of observations with PMAS.

\begin{tabular}{|c|c|c|c|c|c|c|}
\hline Name & Redshift & R.A (2000) & $\delta(2000)$ & WR class & Other designation & Observing date \\
\hline WR 038 & 0.0158 & $17 \mathrm{~h} 29 \mathrm{~m} 06.55 \mathrm{~s}$ & $+56 \mathrm{~d} 53 \mathrm{~m} 19.23 \mathrm{~s}$ & 2 & SHOC 575 & 22-23 Jun 2009 \\
\hline WR 039 & 0.0472 & $17 \mathrm{~h} 35 \mathrm{~m} 01.24 \mathrm{~s}$ & $+57 \mathrm{~d} 03 \mathrm{~m} 08.55 \mathrm{~s}$ & 2 & SHOC 579 & 25 Jun 2009 \\
\hline WR 057 & 0.0179 & $00 \mathrm{~h} 32 \mathrm{~m} 18.59 \mathrm{~s}$ & $+15 \mathrm{~d} 00 \mathrm{~m} 14.16 \mathrm{~s}$ & 3 & SHOC 022 & 11 Oct 2009 \\
\hline WR 266 & 0.0213 & $15 \mathrm{~h} 38 \mathrm{~m} 22.00 \mathrm{~s}$ & $+45 \mathrm{~d} 48 \mathrm{~m} 07.02 \mathrm{~s}$ & 2 & & 24-25 Jun 2009 \\
\hline WR 404 & 0.0220 & $21 \mathrm{~h} 34 \mathrm{~m} 37.80 \mathrm{~s}$ & $+11 \mathrm{~d} 25 \mathrm{~m} \mathrm{10.19s}$ & 2 & CGCG 427-004 & 24-25 Jun 2009 \\
\hline WR 505 & 0.0164 & $16 \mathrm{~h} 27 \mathrm{~m} 51.17 \mathrm{~s}$ & $+13 \mathrm{~d} 35 \mathrm{~m} 13.73 \mathrm{~s}$ & 2 & & 22-23 Jun 2009 \\
\hline
\end{tabular}

models do not predict high $\mathrm{N} / \mathrm{O}$ values in these lowmetallicity galaxies [19], as for $12+\log (\mathrm{O} / \mathrm{H})<8.2$ most of the $\mathrm{N}$ in the ISM has a primary origin, and therefore, its chemical abundance does not depend on the metallicity of the gas, and the expected N/O ratio for closed-box models has a constant value around $\log (\mathrm{N} / \mathrm{O}) \approx-1.5$. However, many of these integrated observations do not allow us to properly relate the excess in some chemical species with their WR content.

To investigate the issue, among others, of the possible connection between the presence of WR stars and the chemical pollution of the surrounding ISM, we have carried out a program to study metal-poor WR galaxies by means of integral field spectroscopy (IFS; [20-22]). Integrated observations, such as long slit or fibers, may fail to correlate the spatial location and distribution of WR features with respect to the physical conditions and the chemical abundances of the ISM as derived from the optical emission lines. Thus, a twodimensional analysis of the ionized material in galaxies helps us to better understand the interplay between the massive stellar population and the ISM. For instance, whether WR stars are a significant contributor to abundance fluctuations on timescales of $t \sim 10^{7} \mathrm{yr}$ and to the formation of high-ionization lines (e.g., He II $4686 \AA$ ) is still an unsolved issue (e.g., $[23,24])$ that can be probed more precisely when applying IFS to nearby galaxies (see [22]).

Thus far, the results coming from WR galaxies studied IFS point to different scenarios, depending on the relative position between the local or extended $\mathrm{N}$ and/or He enrichment and the location(s) of the WR stars. López-Sánchez et al. [25] claimed to find a local $\mathrm{N}$ overabundance associated with WR emission in IC-10. A similar result is found by James et al. [26] for the blue compact dwarf galaxy Haro 11. Monreal-Ibero et al. [27] also find in NGC5253 local peaks of high N/O (see also [28]), but only some of them are associated with WR emission; so this could be indicative of a different timescale between the formation of the WRs and the mixing of the ejected material with the surrounding ISM. A similar scenario is found by Kehrig et al. [22] in Mrk178, where only one out of three detected WR clusters can be associated with an overabundance of $\mathrm{N}$ and He. Finally, in Pérez-Montero et al. [21], the IFS study of N overabundant objects HS0837 + 4717 and Mrk930, also identified as WR galaxies, leads to high values of $\mathrm{N} / \mathrm{O}$ in scales of more than $1 \mathrm{kpc}$, much beyond the power of the observed WR stars to pollute the ISM in these scales and thus pointing to other hydrodynamical processes affecting the chemistry of the gas in these galaxies, such as the infall of metal-poor gas [29]. James et al. [30] also propose a similar scenario for their results of IFS observations of the merging galaxy UM448.

In this work, we extend the sample of low-metallicity WR galaxies studied by means of IFS by six objects selected from the WR galaxy catalog by Brinchmann et al. [7]. The paper is organized as follows. In Section 2, we describe the observed WR galaxies, and we report the IFS observations and data reduction. In Section 3, we present our results, including emission-line maps and derivation of oxygen and nitrogen chemical abundances and their distributions in the observed fields of view. We also describe the measurement of the WR bumps in the observed galaxies. In Section 4, we discuss our results about the chemical pollution of the ISM in the context of WR stars the surrounding ISM. Finally, we summarize our results and present our conclusions in Section 5.

\section{Data Acquisition and Reduction}

2.1. Object Selection and Observations. We obtained IFS data of a sample of six objects selected from the SDSS WR galaxy catalog by Brinchmann et al. [7] following three criteria: (i) galaxies should be associated with a WR index of 2 (convincing WR features in the SDSS spectrum) or 3 (very clear features) in the catalog, (ii) the main ionization source should be dominated by star formation as derived using diagnostic diagrams based on strong emission lines [31], and (iii) galaxies should have oxygen abundances lower than half the solar value $[12+\log (\mathrm{O} / \mathrm{H}) \approx 8.4]$ as derived from the direct method. These criteria were completed with two other observational conditions: (i) to have sizes smaller than the field of view (FoV) of PMAS in lens array mode $16^{\prime \prime} \times 16^{\prime \prime}$ in order to cover the entire galaxy in one single pointing, (ii) all objects were visible from the CAHA observatory in the assigned dates at an air mass lower than 1.2. The six target WR galaxies are listed in Table 1. Column (1) quotes the names of the objects from the catalog of Brinchmann et al. [7]. Column (2) shows the redshift of each galaxy. Columns (3) and (4) give the object coordinates. Column (5) gives the WR index as done by Brinchmann et al. [7], column (6) gives other names, and finally column (7) shows the observing date.

The data were acquired with the Integral Field Unit (IFU) Potsdam Multi-Aperture Spectrophotometer (PMAS), developed at the Astrophysikalisches Institut Potsdam [32]. PMAS is attached to the $3.5 \mathrm{~m}$ Telescope at the CAHA Observatory (Spain). The PMAS spectrograph is equipped with 256 fibers coupled to a $16 \times 16$ lens array. Each fiber has a spatial 
sampling of $1^{\prime \prime} \times 1^{\prime \prime}$ on the sky resulting in a FoV of $16^{\prime \prime} \times 16^{\prime \prime}$ collecting square areas known as spaxels.

We were awarded a service mode run on the nights of June 22-25, 2009 (program F09-3.5-27). In addition, we continued our program with additional time on October 11th, 2009 as part of the commissing run for the new PMAS CCD.

During observations taken in June, 2009 with the old PMAS $2 \mathrm{~K} \times 2 \mathrm{~K} C \mathrm{CD}$, we used the $\mathrm{V} 600$ grating in two separate spectral ranges: the blue side, covering a spectral range $\sim 3700-5200 \AA$ (centered at $4500 \AA$ ), and the red one (centered at $6325 \AA$ ), providing a spectral range from $\sim 5350$ to $6850 \AA$. For the galaxy WR057, taken with the new $4 \mathrm{~K} \times$ $4 \mathrm{~K}$ PMAS CCD but with the same resolution, we were able to cover the whole optical spectral range $(\sim 3700-6850 \AA)$ in one shot using the same V600 grating. The data were binned by a factor of 2 in the spectral direction, yielding a spectral resolution of $\sim 1.6 \AA /$ pixel. The data were acquired under nonphotometric conditions and with a seeing varying between $1^{\prime \prime}$ and $1^{\prime \prime} .5$. To avoid major differential atmospheric refraction (DAR) effects, all expositions were taken at air mass $<1.2$. We used one single pointing for each galaxy, covering in all cases the most intense burst of star formation and its surroundings. Observations of the spectrophotometric standard stars BD + 253941 and PG1633 in the first run and BD +284211 for the calibration of WR057 in the second one were obtained during the observing nights for flux calibration. Bias frames, arc exposures ( $\mathrm{HgNe})$, and spectra of a continuum lamp were taken following the science exposures as part of the PMAS baseline calibrations.

2.2. Data Reduction. For all objects observed with the PMAS $2 \mathrm{~K} \times 2 \mathrm{~K} \mathrm{CCD}$, the first steps of the data reduction were done through the R3D package [33]. We used the P3d tool [34] to perform the basic data reduction of WR057, taken with the new PMAS $2 \mathrm{~K} \times 4 \mathrm{~K}$ CCD. This CCD is read out in four quadrants which have slightly different gains [35]. At the time we observed WR057, P3d was the only software capable to handle the characteristics of the new CCD.

After trimming, combining the four quadrants for WR057 and bias subtraction, the expected locations of the spectra were traced on a continuum-lamp exposure obtained before each target exposure. We extracted the target spectra by adding the signal from the 5 pixels around the central traced pixel (that is, the total object spectrum width). With exposures of $\mathrm{HgNe}$ arc lamps obtained immediately after the science exposures, the spectra were wavelength calibrated.

Fibers have different transmissions that may depend on the wavelength. The continuum-lamp exposures were used to determine the differences in the transmission fiber-to-fiber and to obtain a normalized fiber-flat image, including the wavelength dependence. This step was carried out by running the FIBER-FLAT.PL script from the R3D package. In order to homogenize the response of all the fibers, we divided our wavelength calibrated science images by the normalized fiber flat [33]. Then, to remove cosmic rays, different exposures taken at the same pointing were combined using the IмсомBINE routine in IRAF (IRAF is distributed by the National Optical Astronomical Observatories, which are operated by the Association of Universities for Research in Astronomy, Inc., under cooperative agreement with the National Science Foundation). Flux calibration was performed using the IRAF tasks STANDARD, SENSFUNC, and CALIBRATE. We coadded the spectra of the central fibers of the standard star to create a one-dimensional spectrum that was used to obtain the sensitivity function.

The reduced spectra were contained in a data cube for each object.

\section{Results}

3.1. Line Measurements. The emission-line fluxes on the extracted one-dimensional spectra were measured for each spaxel using a Gaussian fitting over the local position of the continuum. This procedure was done using an automatic routine based on the IRAF task SPLOT. In the case of those lines with a lower signal-to-noise (S/N) (e.g., [O III] $4363 \AA$ and [N II] $6584 \AA$ ), the results from this routine were revised by eye inspection and, if necessary, repeated using a manual measurement.

The adjacent continuum to each line can be affected by the underlying stellar population which can depress the intensity of the Balmer emission lines with stellar absorption wings (e.g., [36]). This stellar absorption was studied for the brightest spaxels by fitting a combination of synthesis spectra of single stellar populations (SSP) libraries by Bruzual and Charlot [37] and the code STARLIGHT (the STARLIGHT project is supported by the Brazilian agencies CNPq, CAPES, and FAPESP and by the France-Brazil CAPES/Cofecub program) $[38,39]$. The fitted spectra were later subtracted from the observed ones and the emission-line intensities of the residuals were compared with the corresponding noncorrected values. For those objects of our sample with very high $\mathrm{H} \beta$ equivalent widths (more than $200 \AA$ for WR039 and around $100 \AA$ for WR038, WR057, WR404, and WR505), the correction at $\mathrm{H} \beta$ wavelength is negligible (less than $1 \AA$ ). Only in the case of WR266, with $\mathrm{EW}(\mathrm{H} \beta)$ of $54 \AA$, we found a correction around $4 \AA$ for $\mathrm{EW}(\mathrm{H} \beta)$. For this object, appropriate corrections for each Balmer line were taken into account according to the SSP fitting in the brightest spaxels.

We calculated the statistical errors of the line fluxes, $\sigma_{l}$, using the expression $\sigma_{l}=\sigma_{c} N^{1 / 2}[1+\mathrm{EW} / N \Delta]^{1 / 2}$ (as in [40]), where $\sigma_{c}$ represents a standard deviation of the noise in a range centred close to the measured emission line, $N$ is the number of pixels used in the measurement of the line flux, EW is the equivalent width of the line, and $\Delta$ is the wavelength dispersion in $\AA /$ pixel. This expression takes into account the error in the continuum and the photon count statistics of the emission line. The error measurements were performed on the extracted one-dimensional spectra. In order to minimize errors in the ratios between a certain emission line and $\mathrm{H} \beta$, we always took first its ratio in relation to the closest hydrogen emission line (i.e., $\mathrm{H} \alpha$ in the case of [N II]), and then, we renormalized using the corresponding theoretical ratio (i.e., at the electron temperature derived in the integrated SDSS observations for each object) from Storey and Hummer [41]. We checked that the variation of this temperature across the FoV of the instrument does not introduce errors in the 
TABLE 2: Total oxygen abundances and N/O derived for the six studied WR galaxies using different methods as described in the text. The confidence level from the Lilliefors test for those spaxels where all involved emission lines were measured is the $P$ value. Column (3) lists the mean value and standard deviation (sigma) from the Gaussian fit as long as $P$ value $>0.05$; otherwise, the mean value and sigma of the data distribution are shown. Column (4) and (5) show the $\mathrm{O} / \mathrm{H}, \mathrm{N} / \mathrm{O}$, and their corresponding errors derived from the brightest spaxel and from the SDSS spectrum for each galaxy, respectively. The number in parenthesis indicates the method used to derive the chemical abundances: (1) from direct method with [O II] $3727 \AA$, (2) from direct method with [O II] 7319, $7330 \AA$, (3) from N2, (4) from O3N2, and (5) from N2O2.

\begin{tabular}{ccccc}
\hline & $P$ value & Mean \pm st. deviation & Brightest & SDSS \\
\hline $\begin{array}{c}\text { 12+ } \log (\mathrm{O} / \mathrm{H}) \\
\text { WR038 }\end{array}$ & & & & \\
WR039 & 0.51 & $8.14 \pm 0.06(3)$ & $8.09 \pm 0.07(1)$ & $8.16 \pm 0.11(2)$ \\
WR057 & 0.07 & $8.06 \pm 0.09(1)$ & $8.96 \pm 0.05(1)$ & $8.13 \pm 0.05(1)$ \\
WR266 & 0.10 & $7.94 \pm 0.06(3)$ & $8.26 \pm 0.30(1)$ & $8.06 \pm 0.06(2)$ \\
WR404 & 0.23 & $8.23 \pm 0.05(3)$ & $8.23 \pm 0.30(4)$ & $8.18 \pm 0.14(1)$ \\
WR505 & 0.02 & $8.26 \pm 0.02(4)$ & $8.16 \pm 0.16(4)$ & $8.23 \pm 0.05(1)$ \\
$\log$ N/O) & 0.30 & $8.31 \pm 0.06(4)$ & & $8.09 \pm 0.08(2)$ \\
WR038 & & & $-0.87 \pm 0.27(1)$ & $-1.06 \pm 0.24(2)$ \\
WR039 & 0.01 & $-1.14 \pm 0.14(5)$ & $-0.80 \pm 0.20(1)$ & $-1.17 \pm 0.14(1)$ \\
WR057 & 0.04 & $-1.09 \pm 0.09(1)$ & $-1.13 \pm 0.30(1)$ & $-1.32 \pm 0.15(2)$ \\
WR266 & 0.39 & $-1.42 \pm 0.07(5)$ & $-1.39 \pm 0.30(5)$ & $-1.18 \pm 0.24(1)$ \\
WR404 & 0.04 & $-1.29 \pm 0.10(5)$ & $-1.15 \pm 0.38(5)$ & $-1.27 \pm 0.16(2)$ \\
WR505 & 0.00 & $-1.37 \pm 0.07(5)$ & & \\
\hline
\end{tabular}

theoretical ratio larger than those associated with the flux of the emission lines.

3.2. Extinction Correction and H $\alpha$ Maps. For each fiber spectrum, we derived its corresponding reddening coefficient, $\mathrm{c}(\mathrm{H} \beta)$, using a weighted fit to the values of the Balmer decrement derived from $\mathrm{H} \alpha / \mathrm{H} \beta$ and $\mathrm{H} \gamma / \mathrm{H} \beta$ as compared to the theoretical values expected for recombination case $\mathrm{B}$ from Storey and Hummer [41] at the electron density and temperature obtained from the integrated SDSS DR-7 spectra and applying the extinction law given by Cardelli et al. [42] with $R_{V}=3.1$. In all cases, homogeneous low values of the reddening constant were derived in agreement with the same values derived from the analysis of the corresponding SDSS one-dimensional spectra.

The fluxes of the emission lines for each spaxel were corrected for extinction using its corresponding $\mathrm{c}(\mathrm{H} \beta)$ value. $\mathrm{H} \alpha$ emission line maps (continuum subtracted and extinction corrected) are shown in Figure 1. As can be seen, the observed FoV encompasses the whole optical extent of our galaxies, which are mostly very compact, with the exception of WR404, which presents a cometary aspect with a low brightness tail towards the NE direction, and WR505, which presents several knots of star formation other than the brightest one at west of the FoV.

3.3. Oxygen and Nitrogen Chemical Abundances. The chemical abundances of oxygen and nitrogen were studied in a representative sample of the observed spaxels in the six WR galaxies using different methods as explained below.

The most accurate method to derive oxygen abundances in emission-line objects, as those in our sample, is the socalled direct method which depends on the relative intensity of both nebular oxygen emission lines to a hydrogen recombination emission line, such as $\mathrm{H} \beta$ (i.e., $\left[\mathrm{O}\right.$ II] $/ \mathrm{H} \beta$ for $\mathrm{O}^{+} / \mathrm{H}^{+}$ and $[\mathrm{O} \mathrm{III}] / \mathrm{H} \beta$ for $\mathrm{O}^{2+} / \mathrm{H}^{+}$), and the previous determination of the electron temperature, via the quotient between auroral-to-nebular emission lines, such as [O III] $4363 \AA$ and [O III] $5007 \AA$ A. See, for instance, Pérez-Montero et al. [21] or Kehrig et al. [22] for additional details of this procedure and how to calculate the low-excitation electron temperature to derive low-excitation ionic abundances. This method was applied to the SDSS DR7 spectra of the brightest regions of the six observed galaxies leading to values of the total oxygen abundances compatible with the low metallicity regime. Owing to the spectral coverage in the SDSS spectra (3800$9100 \AA$ ), the [O II] $3727 \AA$ emission line was not detected in WR038, WR057, and WR505, and the [O II] 7319, $7330 \AA$ were used instead, as described in Kniazev et al. [43]. The total abundances derived in the SDSS spectra using this method are listed in Table 2.

Regarding the PMAS IFS observations, the direct method was applied in a representative number of spaxels only in WR039, where the [O III] $4363 \AA$ emission line was detected with acceptable $\mathrm{S} / \mathrm{N}(>2.5)$. In the other galaxies of our sample, the direct method could only be applied in the brightest spaxels in WR038, WR057, and WR505 and in none of them in WR266 and WR404.

Therefore, the spatial analysis of the chemical properties in these galaxies was done by means of strong-line methods. We first resorted to the N2 parameter (e.g., [44]), defined as the ratio between [N II] $6584 \AA$ and $\mathrm{H} \alpha$. This parameter has the advantage that it does not depend on reddening nor flux calibration uncertainties and is linearly well correlated with oxygen abundance up to solar metallicities. On the contrary, it has an important drawback when it is used for extended 

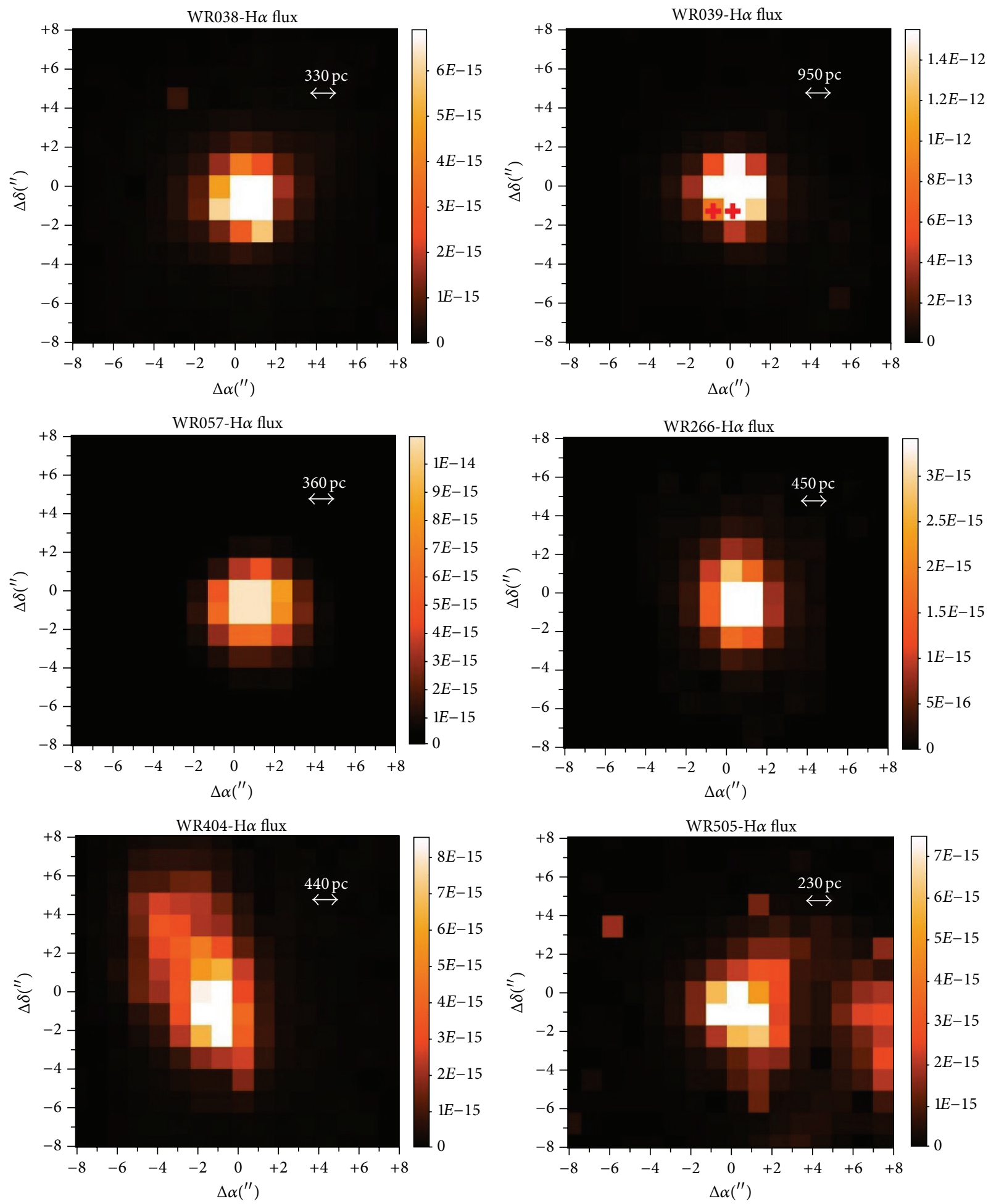

FIGURE 1: Extinction corrected $\mathrm{H} \alpha$ maps of the six observed WR galaxies. In all images, each spaxel has $1^{\prime \prime}$ of resolution. North is up, and east is to the left. Fluxes are in units of erg $/ \mathrm{s} / \mathrm{cm}^{2}$. The relative size in parsecs, at the adopted distance of each object, is indicated. For WR038, the spaxels where the WR bump was detected from our PMAS data are marked with red crosses. 
objects, as those studied in this work by means of IFS, as it also varies as a function of the excitation conditions [45]. We verified in what objects this method could be applied to derive reliably the spatial distribution of the oxygen abundance, by plotting in Figure 2 the relation between the N2 parameter and the $\left[\begin{array}{ll}\mathrm{O} & \mathrm{II}\end{array}\right] /\left[\begin{array}{ll}\mathrm{O} & \mathrm{III}\end{array}\right]$ ratio, which traces the nebular excitation. As can be seen, in the most extended objects of our sample, WR404 and WR505, there is a clear correlation between these two emission-line ratios. In the other objects, there is no clear relation between them, with the possible exception of WR057, but in this object, the spatial variation of N2 is lower than the observational errors. Hence, in the case of WR404 and WR505 galaxies, we used the strong-line parameter $\mathrm{O} 3 \mathrm{~N} 2$, firstly introduced as a metallicity calibrator by Alloin et al. [46] and which is defined as the emission-line ratio between [O III] $5007 \AA$ and [N II] $6584 \AA$. According to several authors, such as Pettini and Pagel [47] or PérezMontero and Contini [48], this parameter is not valid for very low metallicity objects $(12+\log (\mathrm{O} / \mathrm{H})<8.0)$, but, on the contrary, its dependence on excitation is much lower than in the case of N2. According to the values derived from the SDSS spectra for WR404 and WR505, their mean oxygen abundances are higher than the lower limit for O3N2, so this parameter was used for these two objects instead.

For the sake of consistency between the three employed methods (direct method in the case of WR039, N2 parameter for WR038, WR057, and WR266, and O3N2 for WR404 and WR505), we used the calibrations presented in PérezMontero and Contini [48] for N2 and O3N2, which are consistent with the direct method. The resulting oxygen abundance maps of the six observed galaxies are plotted in Figure 3 along with the histogram distributions of the abundances in those spaxels with enough $\mathrm{S} / \mathrm{N}$ in all the involved emission lines $(\mathrm{S} / \mathrm{N}>2.5)$.

In the case of the nitrogen-to-oxygen ratio $(\mathrm{N} / \mathrm{O})$, the direct method can also be used to derive the $\mathrm{N}^{+} / \mathrm{H}^{+}$ratio and then deriving $\mathrm{N} / \mathrm{O}$ using the approximation $\mathrm{N}^{+} / \mathrm{O}^{+} \approx \mathrm{N} / \mathrm{O}$ [as before, see further details in [21, 22]], but this method could only be used in a representative number of spaxels in WR039. For the other five galaxies, we resorted to the $\mathrm{N} 2 \mathrm{O} 2$ parameter, defined as the ratio of [N II] $6584 \AA$ and [O II] $3727 \AA$. This ratio has the advantage that it has a monotonic linear relation with $\mathrm{N} / \mathrm{O}$ and, contrary to $\mathrm{N} 2$, it does not have any dependence on excitation as it only depends on low-excitation emission lines. As in the case of oxygen abundances, we used the empirical calibration of $\mathrm{N} 2 \mathrm{O} 2$ with $\mathrm{N} / \mathrm{O}$ from Pérez-Montero and Contini [48], which is consistent with the direct method derivations of this chemical abundance ratio. The resulting N/O maps of the six observed galaxies are plotted in Figure 4 along with the histogram distributions of the abundances in those spaxels with enough $\mathrm{S} / \mathrm{N}$ in all the involved emission lines.

3.4. Spatial Chemical Homogeneity. To study to what extent the chemical content of the gas can be considered as homogeneous and to give the statistical significance of the $\mathrm{O} / \mathrm{H}$ and N/O distributions, we used the procedure presented in Pérez-Montero et al. [21] and refined in Kehrig et al. [22]. This method is based on the assumption that a certain property can be considered as spatially homogeneous across the observed FoV if two conditions are satisfied: for the corresponding dataset (i) the null hypothesis (i.e., the data come from a normally distributed population) of the Lilliefors test [49] cannot be rejected at the 5\% significance level and (ii) the observed variations of the data distribution around the single mean value can be explained by random errors; that is, the corresponding Gaussian sigma should be lower or of the order of the typical uncertainty of the considered property; we take as typical uncertainty the square root of the weighted sample variance. In Table 2, we show the results from our statistical analysis for both total $\mathrm{O} / \mathrm{H}$ and N/O.

The Lilliefors test for each of the distributions was performed on the linear values of the chemical abundances. The corresponding confidence levels ( $P$ values) are listed in Table 2 with the resulting means and Gaussian sigma in case that the $P$ value is higher than 0.05 . Otherwise, the means and standard deviations are those of the distributions. In those cases where the $P$ value is larger than 0.05 , the second condition imposed to consider a homogeneous distribution is also satisfied in all cases. In all distributions where a strongline method was used to derive both $\mathrm{O} / \mathrm{H}$ and $\mathrm{N} / \mathrm{O}$, the sigma of the Gaussian is much lower than the intrinsic uncertainty associated with these methods ( $\sim 0.3 \mathrm{dex})$. For WR039, where the direct method was used, the weighted sigma for both oxygen abundance and N/O is 0.2 dex, which also is larger than the usual sigmas in the Gaussian fittings.

\section{Discussion: Are WR Stars Able to Pollute the ISM of Galaxies?}

The use of IFS is fundamental to study the spatial extent of the chemical properties of the ISM in extended objects. In this context, this technique along with the use of appropriate statistical tools allows us to explore the presence of chemical inhomogeneities in both $\mathrm{O} / \mathrm{H}$ and $\mathrm{N} / \mathrm{O}$ and to relate this local pollution with the position of WR stars, whose stellar winds enrich the surrounding ISM with the products of the main sequence nuclear burning of massive stars.

Our statistical method points to a high degree of homogeneity in $\mathrm{O} / \mathrm{H}$ across the FoV of the studied galaxies, implying scales of several kpc, with the only exception of WR404, where a possible gradient of metallicity is found. This spatial variation of the metallicity goes from lower values in the brightest region of the galaxy at the SE to slightly higher values in the low surface brightness tail towards the NW.

Our spatial analysis of N/O reveals that only WR057 presents values for which the homogeneity of this abundance ratio cannot be ruled out. In contrast, for all the other cases, the conditions assumed to consider a homogeneous distribution of N/O are not fulfilled, as very high values of this ratio in certain positions of the $\mathrm{FoV}$ were measured.

Although the WR blue bump is detectable in the SDSS spectra of the six selected galaxies, we only found it in our PMAS observations in two spaxels of the WR039 galaxy. These are marked with red crosses in Figure 1. A thorough analysis of the causes of the missing detection of the bump in the other objects will be performed in a forthcoming paper. Figure 5 shows a portion of the spectrum obtained 

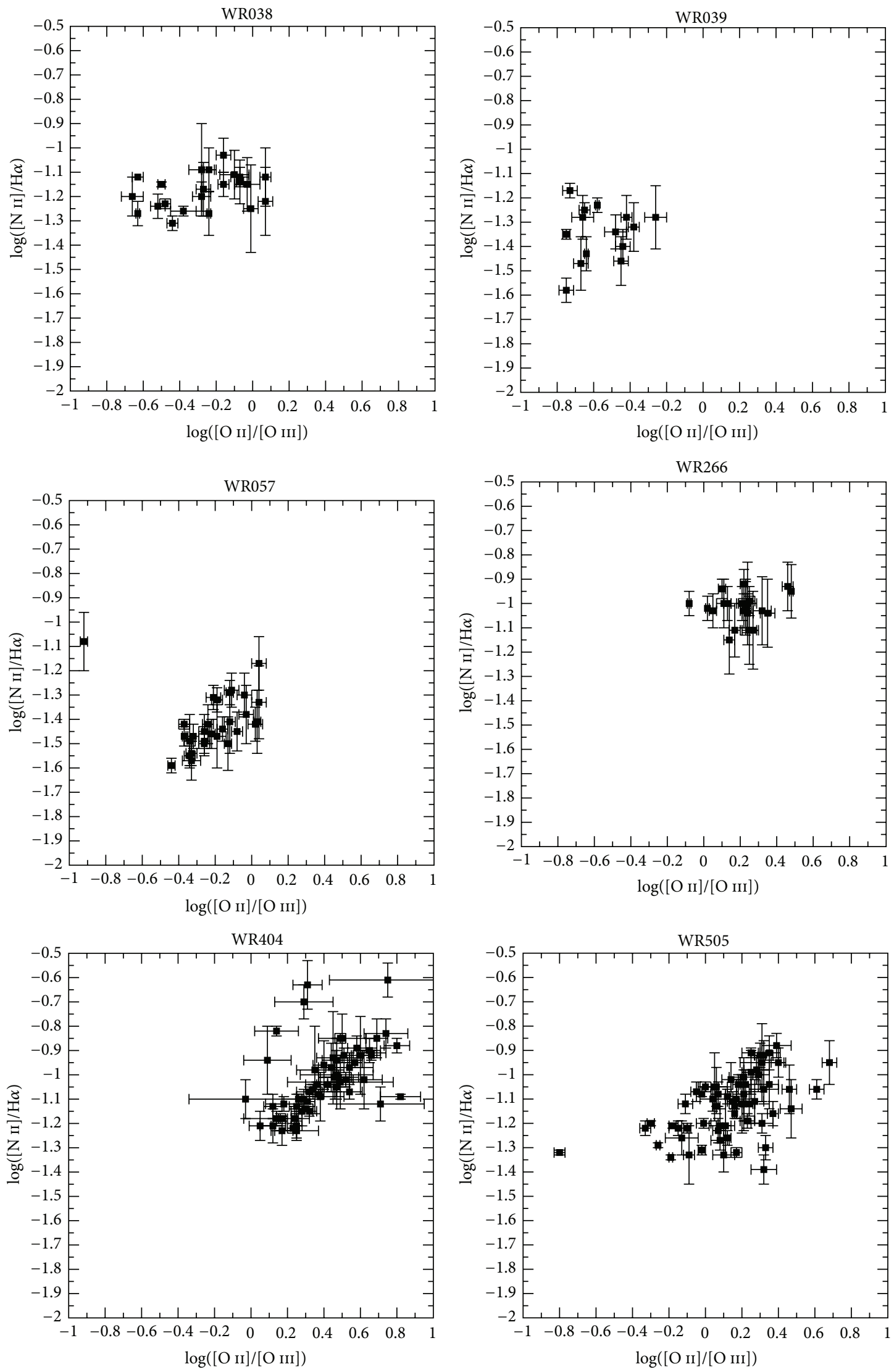

Figure 2: Relation between the $[\mathrm{O}$ II $] /[\mathrm{O}$ III $]$ and $[\mathrm{N} \mathrm{II}] / \mathrm{H} \alpha$ emission line ratios for those spaxels with sufficient $\mathrm{S} / \mathrm{N}$ in the four involved lines of the six observed galaxies. 

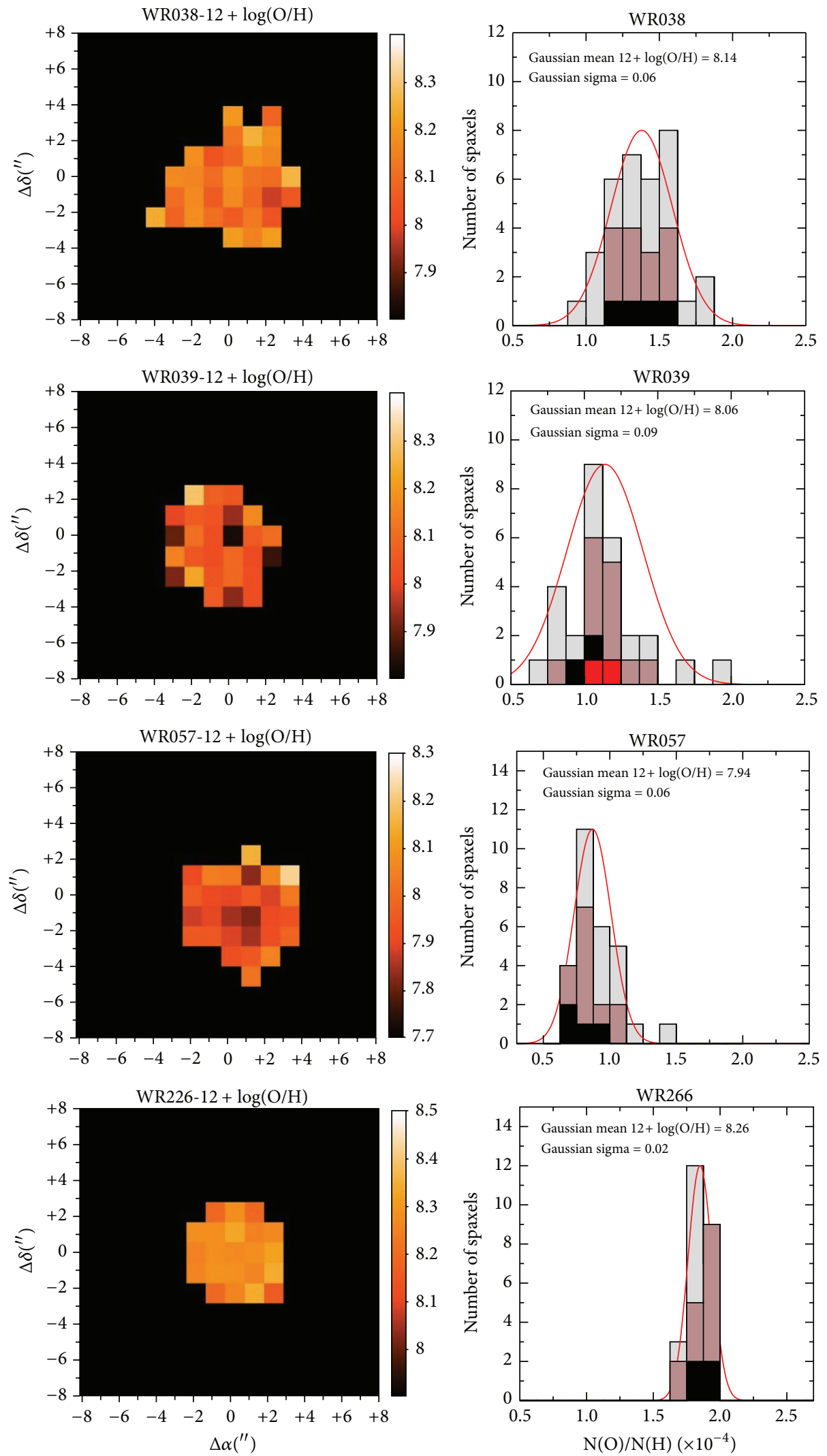

Figure 3: Continued. 

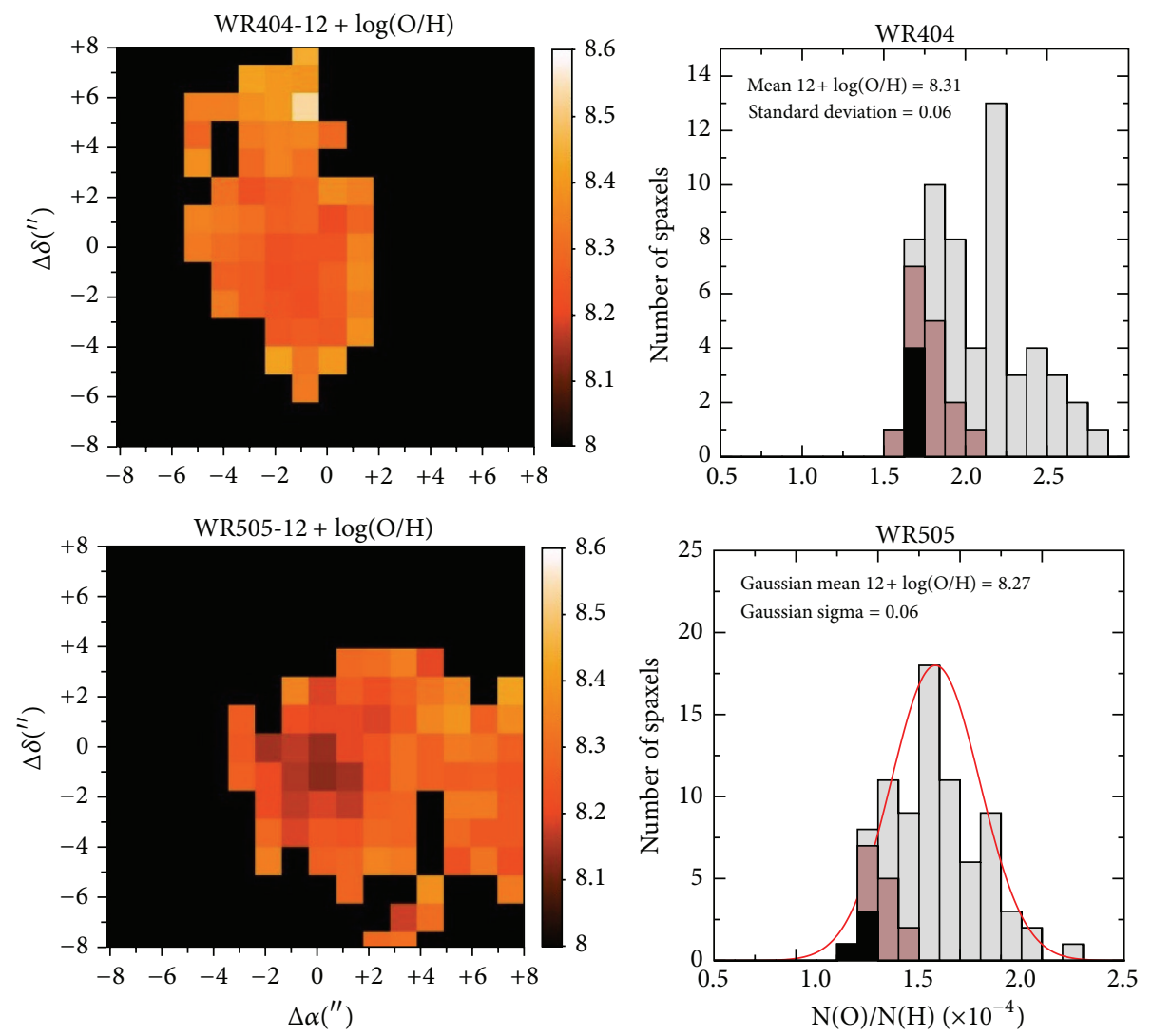

FIGURE 3: Oxygen abundance maps derived as described in the text and histogram distributions in linear scale for WR038, WR039, and WR057. In all images, each spaxel has $1^{\prime \prime}$ resolution, north is up and east is left. The bars in the histogram represent spaxels with detected WR emission (red), spaxels in the same positions as the SDSS pointing (black), spaxels adjacent to this position (brown), and the other spaxels (grey). Same figure for WR266, WR404, and WR505.

by coadding the emission from the 2 spaxels with the WR bump detection. The measured luminosity of the bump at the adopted distance, once the emission lines over the bump were removed, $\left[\mathrm{L}_{\mathrm{WR}}=10^{40.08 \pm 0.08} \mathrm{erg} / \mathrm{s}\right]$, is consistent with the value measured in the integrated SDSS spectrum $\left[\mathrm{L}_{\mathrm{WR}}=\right.$ $\left.10^{39.97 \pm 0.15} \mathrm{erg} / \mathrm{s}\right]$. In contrast, the equivalent width of the bump in these two spaxels $(20 \pm 4 \AA)$ is much higher than in the SDSS spectrum $(8 \pm 4 \AA)$, as expected, taking into account the fact that the collected stellar continuum in the area of the two $1^{\prime \prime}$ PMAS spaxels is fainter than in the $3^{\prime \prime}$ SDSS fiber.

To investigate the possible connection between the detected nitrogen overabundance and the location of the WR stars, we identified in the histograms shown in Figures 3 and 4 the probable positions of the WR stars. In the case of WR039, where the WR bump was detected in our IFS data, the corresponding spaxels with WR emission are plotted as red bars. In the rest of the objects, we selected the four spaxels probably encompassed by the SDSS fiber and which are thought to host the WR population, and we plotted them in the histograms as black bars. This subset of spaxels always includes the brightest $\mathrm{H} \alpha$ position in the observed galaxies. Finally, to study the possible extent of the $\mathrm{N}$ pollution, we also identified the 12 spaxels around these 4 positions of the SDSS fiber as brown bars in the histograms. As can be seen in the histograms and can also be confirmed by visual inspection of the N/O maps, the nitrogen overabundance is tightly related to the position of the WRs in WR038, WR039, WR266, and WR505, although there is not a perfect match between them. This is well illustrated in the unique case where we identified the WR emission, WR039, where the $\mathrm{N}$ overabundance is slightly displaced in relation to the position of the WR bumps. The other galaxy whose N/O is not homogeneous is WR404, but in this case, this is probably related to the gradient of metallicity across its tail detected in the $\mathrm{O} / \mathrm{H}$ analysis, as no direct relation between the $\mathrm{N}$ overabundance and the positions of the WRs is detected.

The results obtained in this work, in which we find evidence for a local nitrogen overabundance (in zones of the order of $100 \mathrm{pc}$ around the position of the WR bumps) in four out of the six observed galaxies by means of IFS have the following implications

(i) WR stars are possibly the main cause of the overabundance of nitrogen observed in 4 out of 6 observed galaxies at spatial scales of the order of several hundreds of parsecs detected around the position of these stars, differing from the objects studied in Pérez-Montero et al. [21], where this overabundance was detected at scales of several kpc. 

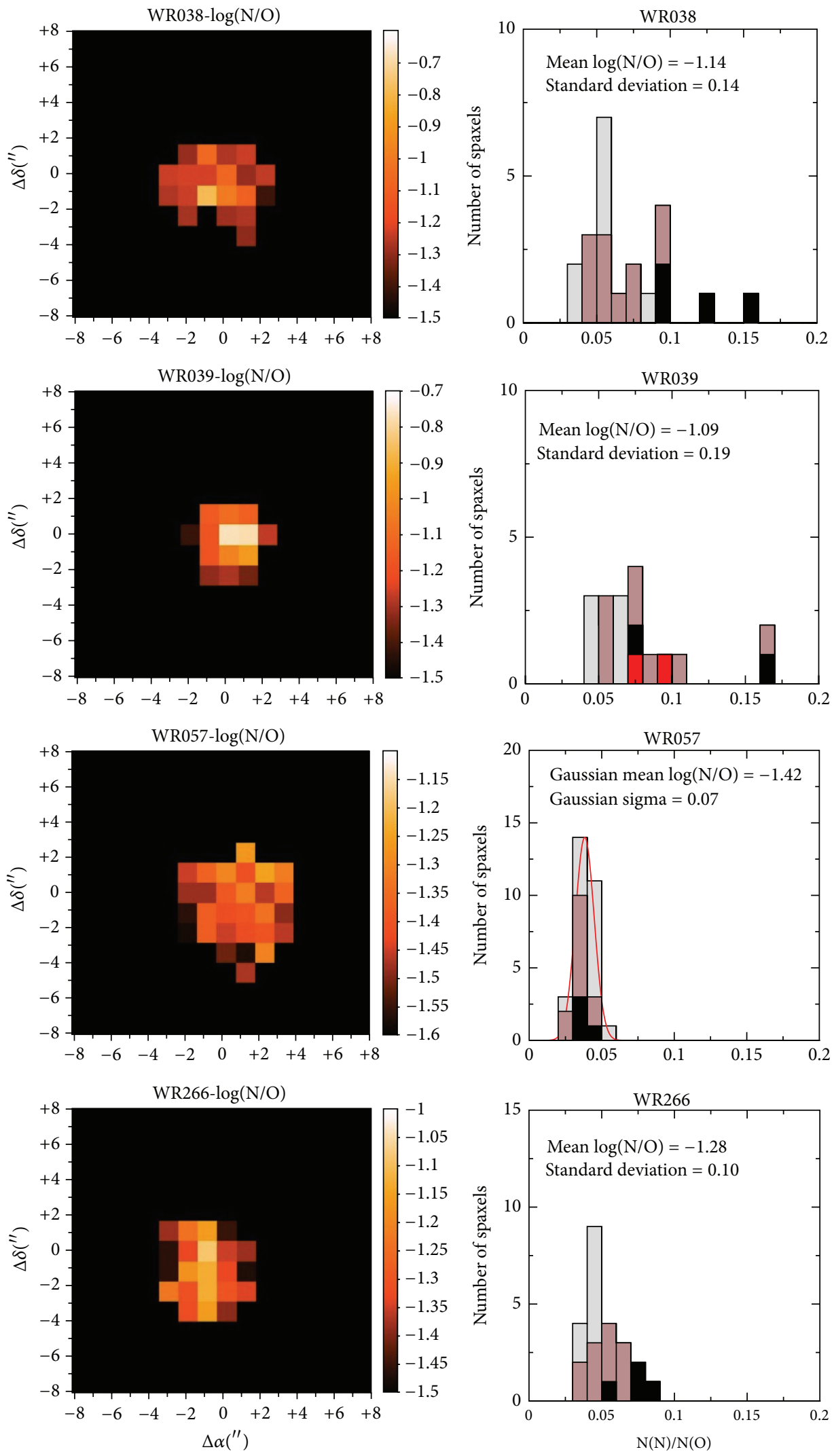

Figure 4: Continued. 

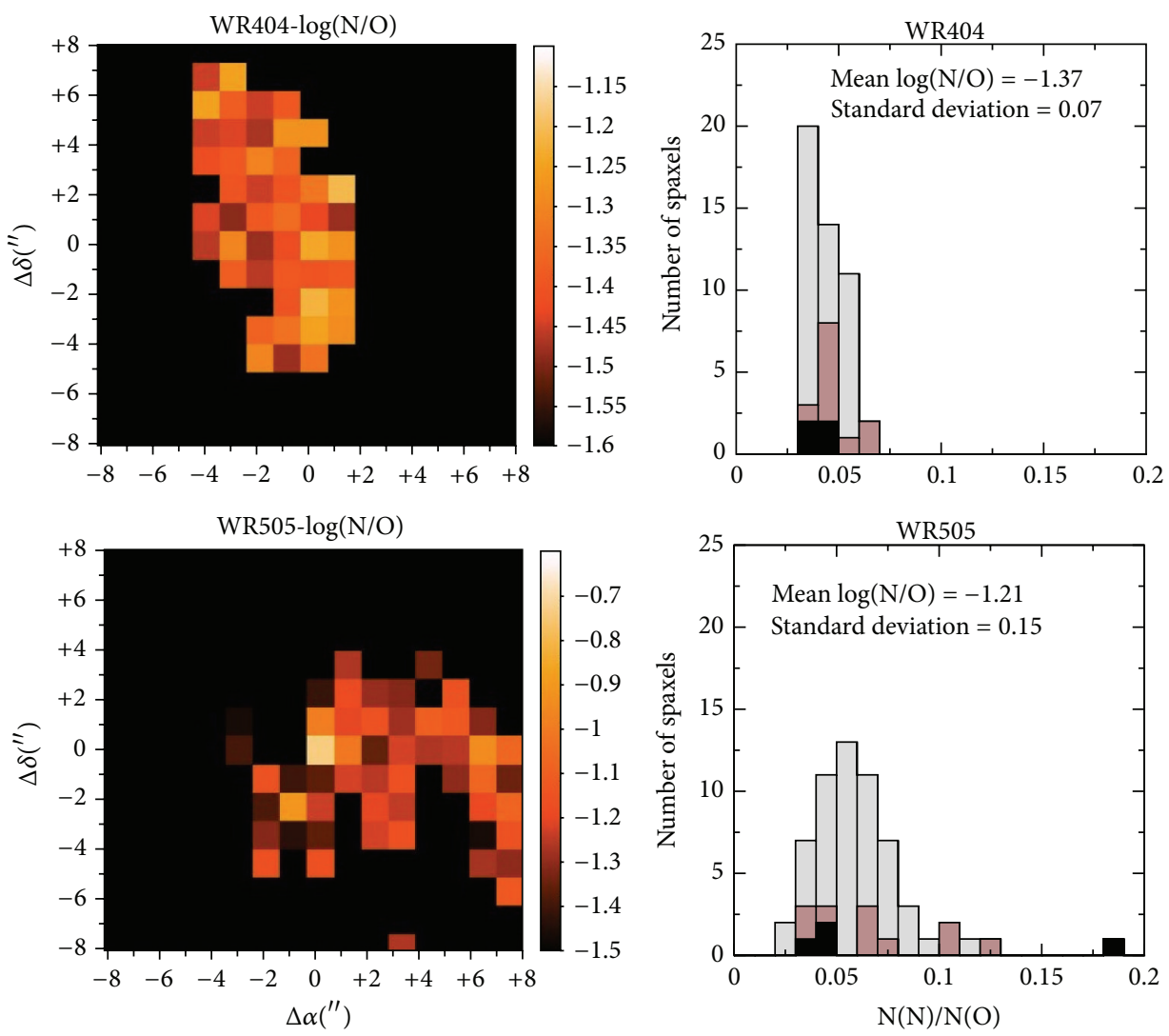

FIGURE 4: N/O ratio maps derived as described in the text and histogram distributions in linear scale for WR038, WR039, and WR057. In all images, each spaxel has $1^{\prime \prime}$ resolution, north is up, and east is left. The colors in the bars have the same meaning as in Figure 3. Same figure for WR266, WR404, and WR505.

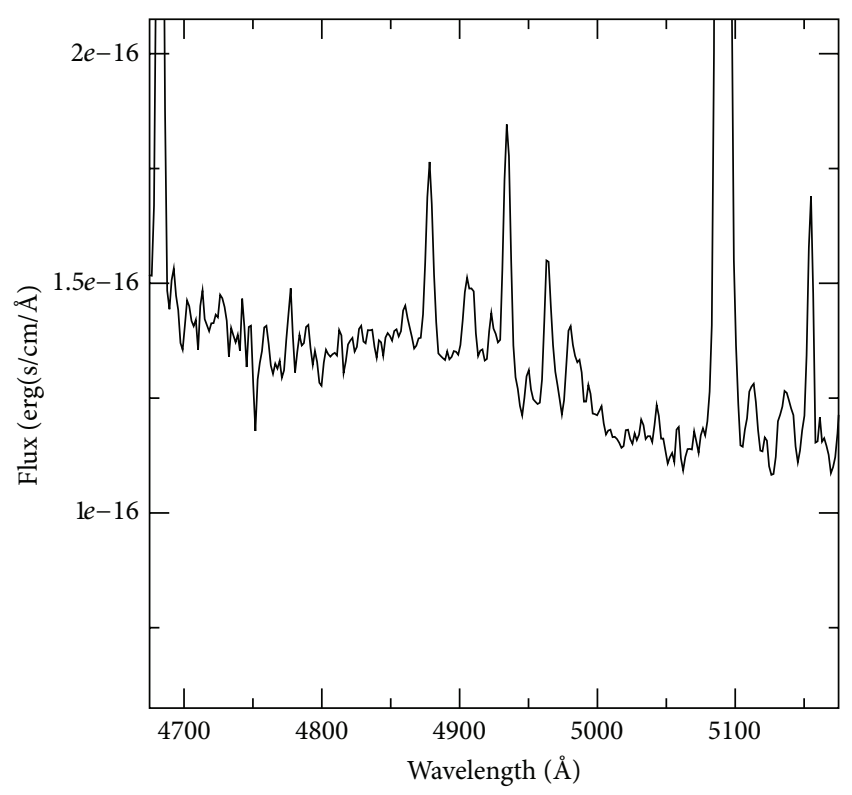

FIGURE 5: Optical spectrum obtained by coadding the two spaxels in WR038 where the Wolf-Rayet blue bump is detected.

According to the chemical yields of massive stars from Mollá and Terlevich [50] presented in Figure 14 of
Pérez-Montero et al. [21], the stellar masses of the ionizing clusters in the sample of WR galaxies studied here (all of them around $10^{7} \mathrm{M}_{\odot}$ ) can produce a N/O excess at distances compatible with the scales at which the $\mathrm{N}$ pollution has been detected by means of IFS in this work.

(ii) As the local $\mathrm{N}$ pollution has not been observed in all the studied objects in this work and in those where it was observed, it does not show a perfect match with the positions of the WR bump. Although it is necessary to take the limited spatial resolution of our observations into account, this mismatch could be possibly due to a timescale offset between the lifetime of the WR stars and the mixing of the ejected material with the ISM. Possibly, the positions with relatively high N/O trace regions where the WR stars were present. In contrast, the positions where the bumps are detected are tracing the ongoing star formation regions. This spatial mismatch between $\mathrm{N}$ excess and WR positions has also been observed in other nearby star-forming objects studied by means of optical IFS (e.g., NGC5253, [27]). This timescale offset between WR lifetimes and $\mathrm{N}$ mixing is supported by the results from other previous works based on IFS data where WR stars were reported, but not the excess 


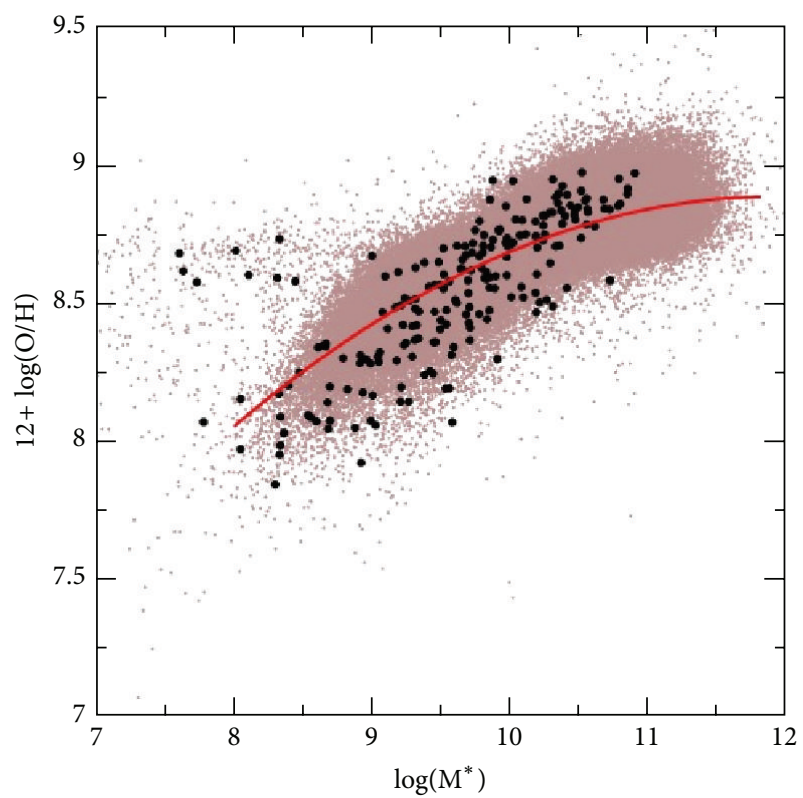

(a)

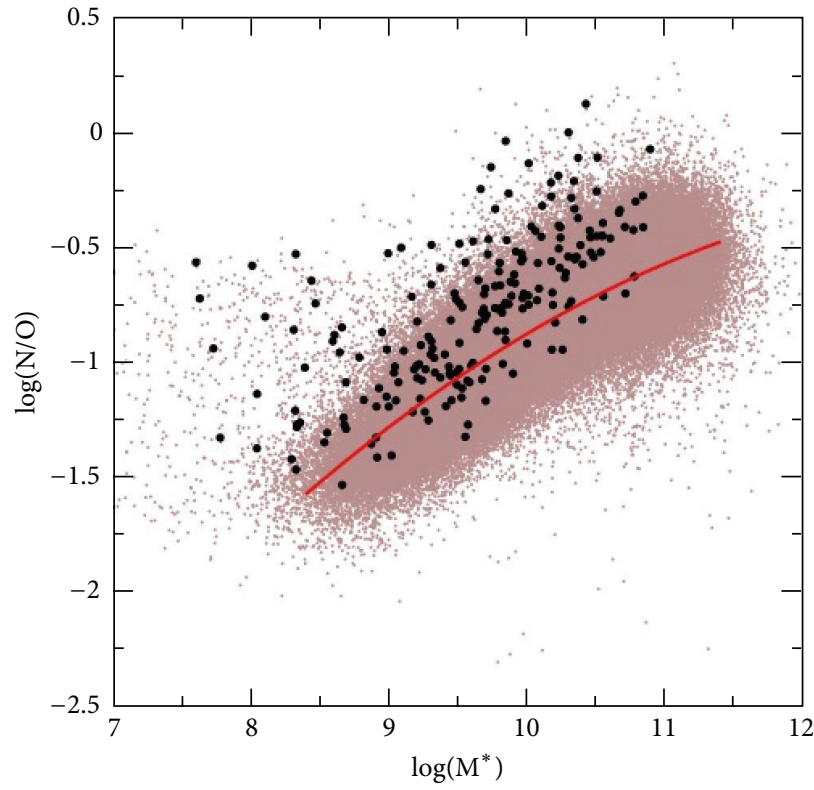

(b)

FIGURE 6: MZR (a) and MNOR (b) relations for the star-forming galaxies selected from the SDSS DR7. The solid red lines, calculated by Pérez-Montero et al. [55], are quadratic fits to the medians in stellar mass bins of $0.2 \mathrm{dex}$. The black points are the galaxies matching the catalog of WR galaxies by Brinchmann et al. [7].

in N/O (e.g., in this work, WR057 and WR404, in HS0837 +4717 and Mrk930 in [21], or in two out of the three WR clusters detected in Mrk178 [22]).

(iii) The local nitrogen pollution happens while homogeneous values of the oxygen abundance are found, which could be indicative that oxygen is not noticeably present in the winds ejected by the WR stars at this stage. Apparently, the properties of these winds (density, velocity, etc.) favour the mixing of their components with the surrounding warm ISM. However, the mixing expected later of the oxygen ejected during the last stages of the WR phase and the subsequent $\mathrm{O}$-rich $\mathrm{SNe}$ explosions have a timescale much longer than that of early WR winds (e.g., [51]).

(iv) Additional mechanisms other than the enrichment due to WR winds are thought to be responsible for the nitrogen overabundance in star-forming galaxies. This is the case of collisional deexcitation of $\mathrm{O}^{+}$in strong shocks associated with mergers [52] or other hydrodynamical processes, such as the infall of pristine gas [29], which can at same time reduce the overall metallicity of galaxies and boost the star formation. These processes could be behind the relation between metallicity and star-formation rate in galaxies. On the contrary, such a mechanism will not have any influence on the abundance ratio of metals as $\mathrm{N}$ and $\mathrm{O}[53]$. A very suitable tool to identify these processes and to distinguish them from local pollution, as in the case of $\mathrm{N}$ ejection by WR stars, is the simultaneous analysis of the relations between stellar mass and metallicity (MZR) and with N/O (MNOR). This was already used by Amorín et al. [54] to understand the low metallicity combined with $\mathrm{N} / \mathrm{O}$ ratio much higher than the values in the plateau of the diagram $\mathrm{O} / \mathrm{H}$ versus $\mathrm{N} / \mathrm{O}$ measured in green pea galaxies. In that work the analysis of these galaxies shows that these objects have the expected N/O for their masses, while they have systematically lower metallicities, even though WR stars have been detected in deep GTC spectra of some of them [18].

Hence, for WR galaxies, we try to understand their average observed N/O excess, as reported by Brinchmann et al. [7], doing the same analysis. In Figure 6 the MZR is shown for the star-forming galaxies of the SDSS, with their stellar masses compiled from the Max Planck Institute for Astrophysics-Johns Hopkins University (MPA-JHU) catalog (available at http://www.mpa-garching.mpg.de/SDSS/) and oxygen abundances calculated using the N2 parameter for emission lines with $\mathrm{S} / \mathrm{N}$ larger than 2 . The solid red line, as explained in Pérez-Montero et al. [55], is a quadratic fit to the medians for stellar mass bins of 0.2 dex. The black points are the matches between the WR galaxy catalog by Brinchmann et al. [7] and all the other star-forming SDSS galaxies selected from the MPA/JHU list. The number of matches obeying the $\mathrm{S} / \mathrm{N}$ criterion and having a minimum redshift $(z>0.02)$ to avoid serious aperture effects in the determination of the stellar mass, as described in Pérez-Montero et al. [55], is 254. In the right panel of the same figure, the MNOR is shown, as calculated using the N2S2 parameter with the calibration by Pérez-Montero and Contini [48]. As can be appreciated and contrary to green pea galaxies, the WR galaxies are in average in agreement with the metallicities expected for their stellar 
mass but, in contrast, have larger N/O ratios, which could be just local pollution values in the same region covered by the SDSS fiber and possibly due to the enrichment by WR stars.

\section{Summary}

In this work, we presented $3.5 \mathrm{~m}$ CAHA-PMAS IFS observations of six metal-poor compact WR galaxies selected from the catalog published by Brinchmann et al. [7] in the optical spectral range 3700-6850 A. Our aim is to study the connection between the presence of WR stars and N/O excess as compared with the values predicted by chemical evolution models at this metallicity regime.

We derived $\mathrm{O} / \mathrm{H}$ and $\mathrm{N} / \mathrm{O}$ abundances ratios using the direct method (i.e., with the determination of the electron temperature) or strong-line methods based on [N II] $6584 \AA$ emission line, such as $\mathrm{N} 2, \mathrm{O} 3 \mathrm{~N} 2$ (for $\mathrm{O} / \mathrm{H}$ ), and $\mathrm{N} 2 \mathrm{O} 2$ (for $\mathrm{N} / \mathrm{O}$ ) with the calibrations provided by Pérez-Montero and Contini [48], which are consistent with the direct method. We studied the homogeneity of the spatial distributions of both $\mathrm{O} / \mathrm{H}$ and $\mathrm{N} / \mathrm{O}$ using the same statistical procedure introduced by Pérez-Montero et al. [21] and improved by Kehrig et al. [22].

Our results indicate that in all the studied objects $\mathrm{O} / \mathrm{H}$ can be considered as uniform in scales of the order of several kpc, with the exception of WR404, for which a gradient of $\mathrm{O} / \mathrm{H}$ is found in the same direction of a low surface brightness tail. In contrast, $\mathrm{N} / \mathrm{O}$ can only be considered as homogeneous in WR057. In four of the six studied galaxies (WR038, WR039, WR266, and WR505), we found positions associated with or close to the WR stars with $\mathrm{N}$ excess in spatial scales of the order of several hundreds of pc. We discussed that, according to the models presented by Pérez-Montero et al. [21] based on massive star yields of Mollá and Terlevich [50], the $\mathrm{N}$ excess length scale is consistent with the distances at which the stellar clusters in these galaxies can enhance the gas-phase abundance of N. On the other hand, our analysis of both the MZR and the MNOR of the WR galaxies of the SDSS WR catalog of Brinchmann et al. [7] excludes hydrodynamical effects, such as metal-poor gas inflows, as the more frequent cause of the $\mathrm{N}$ excess detected in the SDSS galaxies with a detection of the WR bump.

Based on observations collected at the Centro Astronómico Hispano Alemán (CAHA) at Calar Alto, operated jointly by the Max-Planck Institut für Astronomie and the Instituto de Astrofísica de Andalucía (CSIC).

\section{Acknowledgment}

This work has been partially supported by projects AYA200767965-C03-02 and AYA2010-21887-C04-01 of the Spanish National Plan for Astronomy and Astrophysics. The authors thank an anonymous referee for his/her very thorough revision of this paper that has helped to improve it.

\section{References}

[1] D. E. Osterbrock and R. D. Cohen, "Two galaxies with WolfRayet features in their spectra," Astrophysical Journal, vol. 261, pp. 64-69, 1982.
[2] D. A. Allen, A. E. Wright, and W. M. Goss, "The dwarf emission galaxy He 2.10," Monthly Notices of the Royal Astronomical Society, vol. 177, p. 91, 1976.

[3] P. S. Conti, C. D. Garmany, and P. Massey, "Spectroscopic studies of Wolf-Rayet stars. V-Optical spectrophotometry of the emission lines in Small Magellanic Cloud stars," The Astrophysical Journal, vol. 341, pp. 113-119, 1989.

[4] D. Schaerer, T. Contini, and M. Pindao, "New catalogue of WolfRayet galaxies and high-excitation extra-galactic HII regions," Astronomy \& Astrophysics, vol. 136, no. 1, pp. 35-52, 1999.

[5] N. G. Guseva, Y. I. Izotov, and T. X. Thuan, "A spectroscopic study of a large sample of Wolf-Rayet galaxies," Astrophysical Journal Letters, vol. 531, no. 2, pp. 776-803, 2000.

[6] W. Zhang, X. Kong, C. Li, H.-Y. Zhou, and F.-Z. Cheng, "WolfRayet galaxies in the Sloan Digital Sky Survey: the metallicity dependence of the initial mass function," Astrophysical Journal Letters, vol. 655, no. 2 I, pp. 851-862, 2007.

[7] J. Brinchmann, D. Kunth, and F. Durret, "Galaxies with WolfRayet signatures in the low-redshift Universe," Astronomy \& Astrophysics, vol. 485, pp. 657-677, 2008.

[8] P. A. Crowther, "Physical properties of Wolf-Rayet stars," Annual Review of Astronomy \& Astrophysics, vol. 45, pp. 177219, 2007.

[9] F. Legrand, D. Kunth, J.-R. Roy, J. M. Mas-Hesse, and J. R. Walsh, "Detection of WR stars in the metal-poor starburst galaxy IZw 18," Astronomy \& Astrophysics, vol. 326, no. 3, pp. L17-L20, 1997.

[10] R. M. G. Delgado, M. Cerviño, L. P. Martins, C. Leitherer, and P. H. Hauschildt, "Evolutionary stellar population synthesis at high spectral resolution: optical wavelengths," Monthly Notices of the Royal Astronomical Society, vol. 357, no. 3, pp. 945-960, 2005.

[11] J. M. Vílchez and C. Esteban, "Wolf Rayet stars and interrelations with other massive stars in galaxies," in Proceedings of the IAU Symposium, vol. 143, Bali, Indonesia, 1991.

[12] C. Esteban and J. M. Vílchez, "On the chemodynamics of WolfRayet ring nebulae-NGC 6888," The Astrophysical Journal, vol. 390, pp. 536-540, 1992.

[13] A. Fernández-Martín, D. Martín-Gordón, J. M. Vílchez et al., "Ionization structure and chemical abundances of the Wolf-Rayet nebula NGC 6888 with integral field spectroscopy," Astronomy \& Astrophysics, vol. 541, article A119, 2012.

[14] S. Pustilnik, A. Kniazev, A. Pramskij et al., "HS $0837+4717-a$ metal-deficient blue compact galaxy with large nitrogen excess," Astronomy \& Astrophysics, vol. 419, no. 2, pp. 469-484, 2004.

[15] A. R. López-Sánchez, C. Esteban, J. García-Rojas, M. Peimbert, and M. Rodríguez, "The localized chemical pollution in NGC 5253 revisited: results from deep echelle spectrophotometry," The Astrophysical Journal, vol. 656, p. 168, 2007.

[16] G. F. Hägele, E. Pérez-Montero, Á. I. Díaz, E. Terlevich, and R. Terlevich, "The temperature and ionization structure of the emitting gas in H II galaxies: implications for the accuracy of abundance determinations," Monthly Notices of the Royal Astronomical Society, vol. 372, no. 1, pp. 293-312, 2006.

[17] G. F. Hägele, Á. I. Díaz, E. Terlevich, R. Terlevich, E. PérezMontero, and M. V. Cardaci, "Precision abundance analysis of bright H ii galaxies," Monthly Notices of the Royal Astronomical Society, vol. 383, pp. 209-229, 2008.

[18] R. Amorín, E. Pérez-Montero, J. M. Vílchez, and P. Papaderos, "The star formation history and metal content of the green peas. New detailed gtc-osiris spectrophotometry of three galaxies," The Astrophysical Journal, vol. 749, article 185, 2012. 
[19] M. Mollá, J. M. Vílchez, M. Gavilán, and A. I. Díaz, "The nitrogen-to-oxygen evolution in galaxies: the role of the star formation rate," Monthly Notices of the Royal Astronomical Society, vol. 372, pp. 1069-1080, 2006.

[20] C. Kehrig, J. M. Vílchez, and S. F. Sánchez, "The interplay between ionized gas and massive stars in the HII galaxy IIZw70: integral field spectroscopy with PMAS," Astronomy \& Astrophysics, vol. 477, pp. 813-822, 2008.

[21] E. Pérez-Montero, J. M. Vílchez, B. Cedrés et al., "Integral field spectroscopy of nitrogen overabundant blue compact dwarf galaxies," Astronomy \& Astrophysics, vol. 532, article A141, 2011.

[22] C. Kehrig, E. Perez-Montero, J. M. Vílchez et al., "Uncovering multiple Wolf-Rayet star clusters and the ionized ISM in Mrk 178: the closest metal-poor Wolf-Rayet H ii galaxy," Monthly Notices of the Royal Astronomical Society, vol. 432, pp. 27312745, 2013.

[23] C. Kehrig, M. S. Oey, P. A. Crowther et al., "Gemini GMOS spectroscopy of HeII nebulae in M 33," Astronomy \& Astrophysics, vol. 526, no. 16, article A128, 2011.

[24] M. Shirazi and J. Brinchmann, "Strongly star forming galaxies in the local Universe with nebular Heii 4686 emission," Monthly Notices of the Royal Astronomical Society, vol. 421, no. 2, pp. 1043-1063, 2012.

[25] Á. R. López-Sánchez, A. Mesa-Delgado, L. López-Martín, and C. Esteban, "The ionized gas at the centre of IC10: a possible localized chemical pollution by Wolf-Rayet stars," Monthly Notices of the Royal Astronomical Society, vol. 411, no. 3, pp. 2076-2092, 2011.

[26] B. L. James, Y. G. Tsamis, J. R. Walsh, M. J. Barlow, and M. S. Westmoquette, "The Lyman break analogue Haro 11: spatially resolved chemodynamics with VLT FLAMES," Monthly Notices of the Royal Astronomical Society, vol. 430, pp. 2097-2112, 2013.

[27] A. Monreal-Ibero, J. R. Walsh, and J. M. Vílchez, “The ionized gas in the central region of NGC 5253. 2D mapping of the physical and chemical properties," Astronomy \& Astrophysics, vol. 544, article A60, 2012.

[28] M. S. Westmoquette, B. James, A. Monreal-Ibero, and J. R. Walsh, "Piecing together the puzzle of NGC 5253: abundances, kinematics and WR stars," Astronomy \& Astrophysics, vol. 550, article A88, 2013.

[29] J. Köppen and G. Hensler, "Effects of episodic gas infall on the chemical abundances in galaxies," Astronomy \& Astrophysics, vol. 434, no. 2, pp. 531-541, 2005.

[30] B. L. James, Y. G. Tsamis, M. J. Barlow, J. R. Walsh, and M. S. Westmoquette, "The merging dwarf galaxy UM 448: chemodynamics of the ionized gas from VLT integral field spectroscopy," Monthly Notices of the Royal Astronomical Society, vol. 428, no. 1, pp. 86-102, 2013.

[31] J. A. Baldwin, M. M. Phillips, and R. Terlevich, "Classification parameters for the emission-line spectra of extragalactic objects," Publications of the Astronomical Society of the Pacific, vol. 93, pp. 5-19, 1981.

[32] M. M. Roth, T. Fechner, D. Wolter et al., "Commissioning of the CCD231 $4 \mathrm{~K} \times 4 \mathrm{~K}$ detector for PMAS," in High Energy, Optical, and Infrared Detectors for Astronomy IV, vol. 7742 of Proceedings of SPIE, June 2010.

[33] S. F. Sánchez, "Techniques for reducing fiber-fed and integral-field spectroscopy data: An implementation on R3D," Astronomische Nachrichten, vol. 327, pp. 850-861, 2006.

[34] C. Sandin, T. Becker, M. M. Roth et al., "P3D: a general datareduction tool for fiber-fed integral-field spectrographs," Astronomy \& Astrophysics, vol. 515, article A35, 2010.
[35] A. Kelz, M. A. W. Verheijen, M. M. Roth et al., "PMAS: the potsdam multi-aperture spectrophotometer-II. The wide integral field unit PPak," Publications of the Astronomical Society of the Pacific, vol. 118, no. 839, pp. 129-145, 2006.

[36] A. I. Diaz, "Hydrogen absorption line profiles of ionizing star clusters," Monthly Notices of the Royal Astronomical Society, vol. 231, pp. 57-67, 1988.

[37] G. Bruzual and S. Charlot, "Stellar population synthesis at the resolution of 2003," Monthly Notices of the Royal Astronomical Society, vol. 344, no. 4, pp. 1000-1028, 2003.

[38] R. Cid Fernandes, Q. Gu, J. Melnick et al., "The star formation history of Seyfert 2 nuclei," Monthly Notices of the Royal Astronomical Society, vol. 355, no. 1, pp. 273-296, 2004.

[39] A. Mateus, L. Sodré Jr., R. C. Fernandes, G. Stasińska, W. Schoenell, and J. M. Gomes, "Semi-empirical analysis of Sloan Digital Sky Survey galaxies-II. The bimodality of the galaxy population revisited," Monthly Notices of the Royal Astronomical Society, vol. 370, no. 2, pp. 721-737, 2006.

[40] E. Pérez-Montero and A. I. Díaz, "Line temperatures and elemental abundances in H II galaxies," Monthly Notices of the Royal Astronomical Society, vol. 346, no. 1, pp. 105-118, 2003.

[41] P. J. Storey and D. G. Hummer, "Recombination line intensities for hydrogenic ions-IV. Total recombination coefficients and machine-readable tables for $\mathrm{Z}=1$ to 8," Monthly Notices of the Royal Astronomical Society, vol. 272, pp. 41-48, 1995.

[42] J. A. Cardelli, G. C. Clayton, and J. S. Mathis, "The relationship between infrared, optical, and ultraviolet extinction," The Astrophysical Journal, vol. 345, pp. 245-256, 1989.

[43] A. Y. Kniazev, E. K. Grebel, L. Hao, M. A. Strauss, J. Brinkmann, and M. Fukugita, "Discovery of eight new extremely metal-poor galaxies in the sloan digital sky survey," Astrophysical Journal Letters, vol. 593, no. 2, pp. L73-L76, 2003.

[44] G. Denicoló, R. Terlevich, and E. Terlevich, "New light on the search for low-metallicity galaxies-I. The N2 calibrator," Monthly Notices of the Royal Astronomical Society, vol. 330, no. 1, pp. 69-74, 2002.

[45] E. Pérez-Montero and A. I. Díaz, "A comparative analysis of empirical calibrators for nebular metallicity," Monthly Notices of the Royal Astronomical Society, vol. 361, no. 3, pp. 1063-1076, 2005.

[46] D. Alloin, S. Collin-Souffrin, M. Joly, and L. Vigroux, "Nitrogen and oxygen abundances in galaxies," Astronomy \& Astrophysics, vol. 78, pp. 200-216, 1979.

[47] M. Pettini and B. E. J. Pagel, "[O III]/[N II] as an abundance indicator at high redshift," Monthly Notices of the Royal Astronomical Society, vol. 348, no. 3, pp. L59-L63, 2004.

[48] E. Pérez-Montero and T. Contini, "The impact of the nitrogento-oxygen ratio on ionized nebula diagnostics based on [N ii] emission lines," Monthly Notices of the Royal Astronomical Society, vol. 398, no. 2, pp. 949-960, 2009.

[49] H. W. Lilliefors, "On the Kolmogorov-Smirnov test for normality with mean and variance unknown," Journal of the American Statistical Association, vol. 62, pp. 399-402, 1967.

[50] M. Mollá and R. Terlevich, "Modelling the composition of a young star cluster ejecta," Monthly Notices of the Royal Astronomical Society, vol. 425, pp. 1696-1708, 2012.

[51] G. Tenorio-Tagle, "Interstellar matter hydrodynamics and the dispersal and mixing of heavy elements," Astronomical Journal, vol. 111, no. 4, pp. 1641-1650, 1996.

[52] J. C. Raymond, "Shock waves in the interstellar medium," Astrophysical Journal, vol. 39, pp. 1-27, 1979. 
[53] M. G. Edmunds, "General constraints on the effect of gas flows in the chemical evolution of galaxies," Monthly Notices of the Royal Astronomical Society, vol. 246, p. 678, 1990.

[54] R. O. Amorín, E. Pérez-Montero, and J. M. Vílchez, "On the oxygen and nitrogen chemical abundances and the evolution of the "green pea" galaxies", The Astrophysical Journal Letters, vol. 715, article L128, 2010.

[55] E. Pérez-Montero, T. Contini, F. Lamareille et al., "The cosmic evolution of oxygen and nitrogen abundances in star-forming galaxies over the past $10 \mathrm{Gyr}$," Astronomy \& Astrophysics, vol. 549, article A25, 2013. 

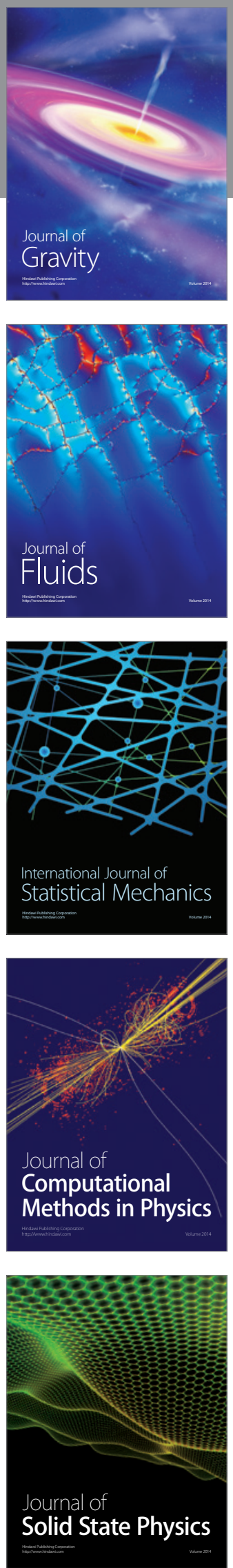

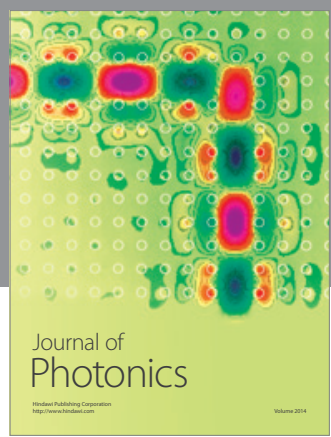

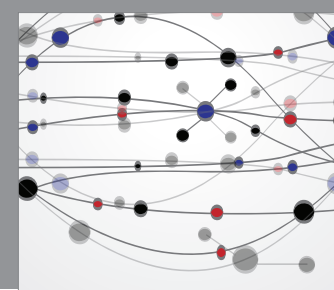

The Scientific World Journal

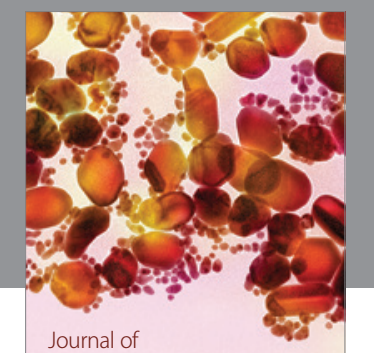

Soft Matter
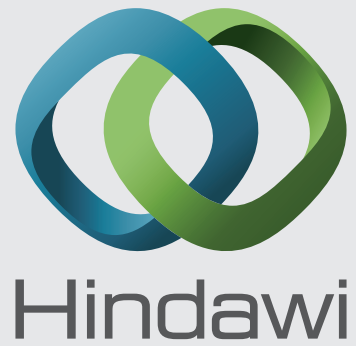

Submit your manuscripts at

http://www.hindawi.com
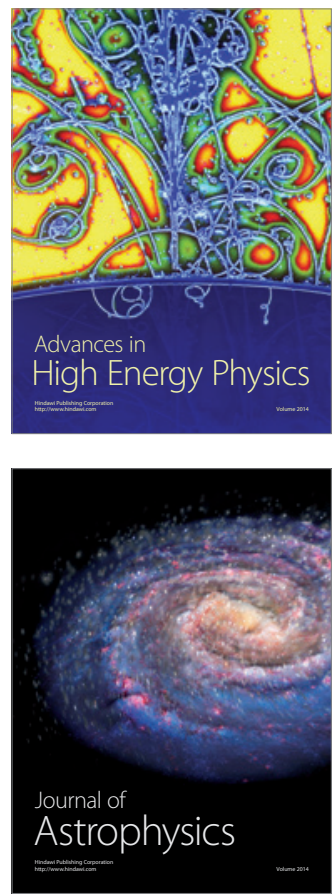
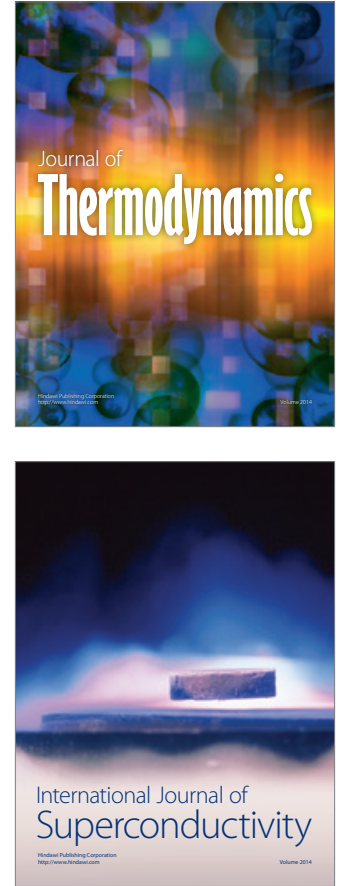
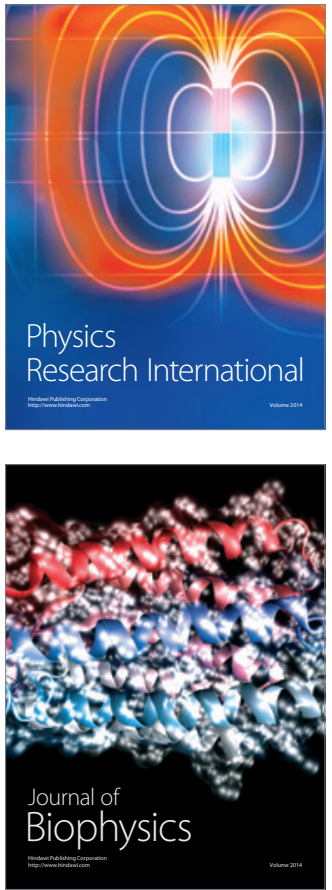
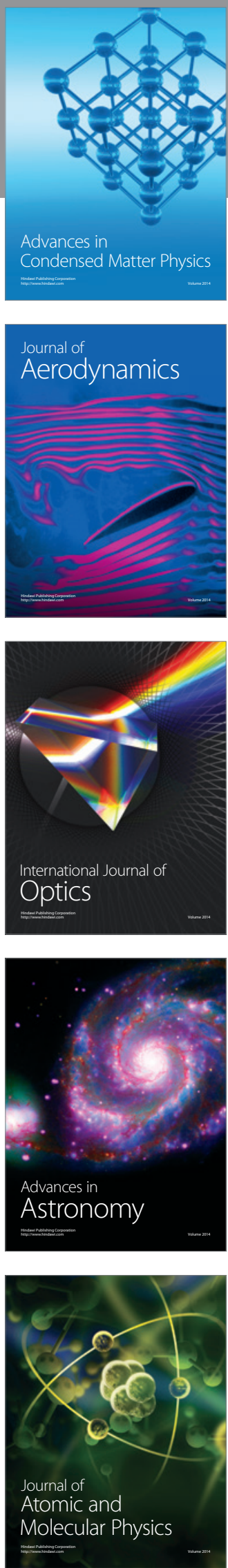